\title{
The expected number of zeros of continuous stationary Gaussian processes*
}

Dedicated to Professor Y. Akizuki for his sixtieth birthday

By

Kiyosi Iто

(Received Jan. 16, 1964)

\section{Introduction}

Let $x(t)=x(t, \omega), \omega \in \Omega(\mathscr{B}, P)$ be a stationary Gaussian process with continuous sample paths. Then the mean $a=E[x(t)]$ is independent of $t$ and the covariance function $r(t)=E[(x(s+t)-a)(x(s)$ $-a)]$ is an even function of $t$, independent of $s$, expressible in the form

$$
r(t)=\int_{-\infty}^{\infty} e^{i \lambda t} d F(\lambda)
$$

with a bounded measure $d F$ symmetric with respect to 0 .

Let $N=N(\omega)$ be the number of zeros of the sample path of $x(t)$ in $0<t<T$ and $N_{c}=N_{c}(\omega)$ the number of crossings of the level $c$ by the sample path of $x(t, \omega)$.

The purpose of this paper is to prove

\section{Theorem.}

$$
E(N)=E\left(N_{c}\right)=\frac{T}{\pi} \sqrt{-\frac{r^{\prime \prime}(0)}{r(0)}} \exp \left(-\frac{a^{2}}{2 r(0)}\right)
$$

where $r^{\prime \prime}(0)$ is the second Schwarz derivative, i.e.,

\footnotetext{
* This work was supported in part by the National Science Foundation, Grant 16319, and in part by the Office of Naval Research, Contract Nonr 225 (28) at Stanford University.
} 


$$
r^{\prime \prime}(0)=\lim _{h \downarrow 0} \frac{r(h)-2 r(0)+r(-h)}{h^{2}} \quad\left(=-\int_{-\infty}^{\infty} \lambda^{2} d F(\lambda)\right) .
$$

$r^{\prime \prime}(0)$ is finite or $-\infty$ according as $\int_{-\infty}^{\infty} \lambda^{2} d F(\lambda)<\infty$ or $=\infty$; in the latter case (2) shows that $E(N)=E\left(N_{c}\right)=\infty$.

Given a constant $a$ and a function $r(t)$ in the form (1), there exists essentially a unique separable stationary process with the mean 0 and the covariance fouction $r(t)$. If Hunt's condition [2]:

$$
\int_{-\infty}^{\infty}[\log (1+|\lambda|)]^{1+\infty} d F(\lambda)<\infty \quad \text { for some } \alpha>0,
$$

is fulfilled, then almost all sample functions of $x(t, \omega)$ are continuous and so (2) holds.

Let us give a historical account of the formula (2).

In 1944 S. O. Rice [1] (see pp. 271-3) proved (2) in case $F(\lambda)$ increases only with a finite number of jumps, i.e.,

$$
r(t)=\sum_{n=1}^{m} e^{i \lambda} n^{t} \sigma_{n} .
$$

Noticing that $\left(x(t), x^{\prime}(t)\right)$ is Gauss distributed with

$$
\left\{\begin{aligned}
E(x(t))=a, & E\left(x^{\prime}(t)\right)=0 \\
E\left(x(t)^{2}\right) & =r(0), \quad E\left(x^{\prime}(t)^{2}\right)=-r^{\prime \prime}(0) \\
& E\left(x(t) x^{\prime}(t)\right)=r^{\prime}(0)=0
\end{aligned}\right.
$$

we have

$$
\begin{aligned}
& E(N)=\int_{0}^{T} E(N(d t)) \quad(N(d t)=\text { the number of zeros of } x(t) \\
& \sim \int_{0}^{T} P(N(d t)=1) \\
& \sim \int_{0}^{T} \int_{0}^{\infty} P\left(x^{\prime}(t) \in d \eta,-\eta d t<x(t)<0\right) \\
& +\int_{0}^{T} \int_{-\infty}^{0} P\left(x^{\prime}(t) \in d \eta, 0<x(t)<\eta d t\right) \\
& =2 \int_{0}^{T} \int_{0}^{\infty} \frac{1}{2 \pi \sqrt{-r^{\prime \prime}(0)} \boldsymbol{r ( 0 )}} \exp \left\{-\frac{(0-a)^{2}}{2 r(0)}-\frac{\eta^{2}}{2\left(-r^{\prime \prime}(0)\right)}\right\} \eta d \eta d t \\
& =\frac{T}{\pi} \sqrt{-\frac{r^{\prime \prime}(0)}{r(0)}} \exp \left\{-\frac{a^{2}}{2 r(0)}\right\} \text {. }
\end{aligned}
$$

This is the outline of Rice's proof. 
In 1957 U. Grenander and M. Rosenblatt [3] (see p. 271-3) gave a neater proof to the formula in the same special cace using the following formula on the number $N$ of zeros of a function $f(t)$ due to M. Kac [4]:

$$
N \sim \frac{1}{2 \varepsilon} \int_{0}^{T}\left|f^{\prime}(t)\right| e_{(-\varepsilon, \varepsilon)}(f(t)) d t
$$

where $e_{(-\varepsilon, \varepsilon)}$ is the indicator function of the interval $(-\varepsilon, \varepsilon)$.

There were some technical gaps in both the proof of Rice and that of Grenander-Rosenblatt in connection with the estimation and interchangeability of the limit and the expectation.

In $1960 \mathrm{~V}$. A. Ivanov [5] gave the first rigorous proof to the formula in cace

$$
\int_{-\infty}^{\infty} \lambda^{4} d F(\lambda)<\infty
$$

carrying out the argument of Grenander-Rosenblatt carefully.

On 1961 E. V. Bulinskaya [6] proved it in a more general case

$$
\int_{-\infty}^{\infty} \log (1+|\lambda|)^{1+\alpha} \lambda^{2} d F(\lambda)<\infty \quad \text { for some } \alpha>0 .
$$

She used a lower estimate of the number of zeros of $f(t)$ :

$$
\begin{aligned}
N \sim \lim _{p \rightarrow \infty} \sum_{q=1}^{2^{p}} c\left(f\left(\frac{(q-1) T}{2^{p}}\right), f\left(\frac{q T}{2^{p}}\right)\right) \\
(c(\xi, \eta)=1 \text { or } 0 \text { according as } \xi \eta<0 \text { or } \geq 0)
\end{aligned}
$$

in addition to the estimate (8) which is actually an upper one.

Both Ivanov and Bulinskaya imposed the conditions ((9), (10)) to let almost all sample functions be continuously differential, but a slight modification of their methods will enable us to prove the formula in complete generality, as we can see in our paper.

We wish to express our thanks to Professor J. Chover who gave us a lot of information on this subject.

\section{The upper and lower bounds of the number of zeros}

Let $n$ denote the number of zeros of $f(t)$ in $0<t<T$ and $n_{c}$ the number of crossings of the level $c$ by $f(t)$. The numbers $n$ and $n_{c}$ may be finite but $n \geq n_{c}$ is clear in any case. 
The following lemmas will be used later.

Lemma 1. If $f(t)$ is continuous, then

$$
n \geq n_{c} \geq \lim _{p \rightarrow \infty} \sum_{q=1}^{2^{p}} c\left(f\left(\frac{(q-1) T}{2^{p}}\right), f\left(\frac{q T}{2^{p}}\right)\right) \quad \text { (monotone limit) }
$$

where $c(\xi, \eta)$ is the indicator function of the two-dimensional region: $\xi_{\eta}<0$.

Lemma 2. If $f(t)$ is absolutely continuous and vanishes only on a t-set which contains no interval, then

$$
n_{c} \leq n \leq \lim _{\varepsilon \downarrow 0} \frac{1}{2 \varepsilon} \int_{0}^{T}\left|f^{\prime}(t)\right| e_{(-\varepsilon, \mathrm{z})}(f(t)) d t,
$$

where $e_{(-\varepsilon, 8)}(\xi)$ is the indicator function of the interval $(-\varepsilon, \varepsilon)$.

Proof. Lemma 1 is evident by the intermediate value theorem for continuous functions.

To prove Lemma 2 , it is enough to show that

$$
\lim _{\varepsilon} \frac{1}{2 \varepsilon} \int_{0}^{T}\left|f^{\prime}(t)\right| e_{(-\varepsilon, \varepsilon)}(f(t)) d t \geq m
$$

for any finite number $m \leq n$. If $m \leq n$, we can take a sequence of zeros $t_{1}<t_{2}<\cdots<t_{m}$ of $f(t)$ in $0<t<T$. Let $u_{i}=u_{i}(\varepsilon)$ be the greatest number $u<t_{i}$ with $|f(u)|=\varepsilon\left(u_{i}=-\infty\right.$ if there exists no such $u)$ and $v_{i}=v_{i}(\varepsilon)$ be the least number $v<t_{i}$ with $|f(v)|=\varepsilon$ $\left(v_{i}=\infty\right.$ if there exists no such $\left.v\right)$. Then we have some $\varepsilon_{0}>0$ such that, for any positive $\varepsilon<\varepsilon_{0}$,

$$
0<u_{1}<t_{1}<v_{1}<u_{2}<t_{2}<v_{2}<\cdots<u_{m}<t_{m}<v_{m}<T ;
$$

if otherwise, $f(t)$ would vanish on a subinterval of $(0, T)$ in contradiction with our assumption. It follows from (3) that

$$
\begin{aligned}
& \frac{1}{2 \varepsilon} \int_{0}^{T}\left|f^{\prime}(t)\right| e_{(-8,8)}(f(t)) d t \\
& \quad \geq \frac{1}{2 \varepsilon} \sum_{i=1}^{m}\left[\int_{u_{i}}^{t_{i}}\left|f^{\prime}(t)\right| d t+\int_{t_{i}}^{n_{i}}\left|f^{\prime}(t)\right| d t\right] \\
& \quad \geq \frac{1}{2 \varepsilon} \sum_{i=1}^{m}\left[\left|f\left(t_{i}\right)-f\left(u_{i}\right)\right|+\left|f\left(v_{i}\right)-f\left(t_{i}\right)\right|\right]=m,
\end{aligned}
$$

which completes the proof. 
3. Proof of Theorem in case $\int_{-\infty}^{\infty} \lambda^{2} d F(\lambda)<\infty$.

We shall consider a stationary Gaussian process described in the introduction and assume that

$$
\int_{-\infty}^{\infty} \lambda^{2} d F(\lambda)<\infty
$$

We shall prove several preliminary propositions.

Proposition 1. $x(t)$ is differentiable at every time point $t$ in the sense of limit in the mean, i.e., limit with respect to the norm in $L^{2}(\Omega, \mathscr{B}, P)$.

By our assumption we have

$$
\begin{aligned}
& \| \frac{x(t+h)-x(t)}{h}-\left.\frac{x(t+k)-x(t)}{k}\right|^{2} \\
& \quad=\int_{-\infty}^{\infty}\left|\frac{e^{i \lambda h}-1}{h}-\frac{e^{i \lambda k}-1}{k}\right|^{2} d F(\lambda) \rightarrow 0 \quad(h, k \rightarrow 0)
\end{aligned}
$$

since the integrand tends to 0 and is bounded by $4 \lambda^{2}$.

Proposition 2. $x(t), x^{\prime}(t), t \in[-\infty, \infty)$ form a Gaussian system with

$$
\left\{\begin{array}{c}
E(x(t))=n, \quad E\left(x^{\prime}(t)\right)=0 \\
E((x(t)-a)(x(s)-a))=r(t-s)=\int e^{i \lambda(t-s)} d F(\lambda) \\
E\left(x^{\prime}(t)(x(s)-a)\right)=r^{\prime}(t-s)=\int e^{i \lambda(t-s)} i \lambda d F(\lambda) \\
E\left(x^{\prime}(t) x^{\prime}(s)\right)=-r^{\prime \prime}(t-s)=\int e^{i \lambda(t-s)} \lambda^{2} d F(\lambda) .
\end{array}\right.
$$

This is clear, because $x(t), t \in(-\infty, \infty)$ is a Gaussian system and $x^{\prime}(t)$ belongs to the closed linear subspace of $L^{2}(\Omega, \mathscr{B}, P)$ spanned by $x(t), t \in(-\infty, \infty)$.

Proposition 3. $x^{\prime}(t)$ has a measurable version, namely we can construct a function $y(t, \omega)$ measurable in $(t, \omega)$ such that $P\left(x^{\prime}(t)=y(t)\right)$ $=1$ for every $t$.

Write $y_{h}(t)$ for $h^{-1}(x(t+h)-x(t)) . \quad y_{h}(t, \omega)$ is measurable in $(t, \omega)$ 
because it is measurable in $\omega$ for each value of $t$ and continuous in $t$ for almost every value of $\omega$. It follows from Proposition 2 that

$$
\left\|y_{h}(t)-x^{\prime}(t)\right\|^{2}=\int_{-\infty}^{\infty}\left|\frac{e^{i \lambda h}-1}{h}-i \lambda\right|^{2} d F(\lambda) \leq \frac{h^{2}}{4} \int_{-\infty}^{\infty} \lambda^{2} d F(\lambda) .
$$

Writing $y_{n}(t)$ for $y_{2^{-n}}(t)$, we have, for every $t$,

$$
\left\|y_{n}(t)-x^{\prime}(t)\right\|^{2} \leq 2^{-2 n-2} \int_{-\infty}^{\infty} \lambda^{2} d F(\lambda)
$$

and so

$$
P\left(y_{n}(t) \rightarrow x^{\prime}(t) \text { as } \quad n \rightarrow \infty\right)=1,
$$

i.e.

$$
P\left(\lim _{n \rightarrow \infty} y_{n}(t)=x^{\prime}(t)\right)=1 .
$$

Thus $y(t) \equiv \varlimsup_{n \rightarrow \infty} y_{n}(t)$ is what we wanted to construct.

From now on we shall denote the measurable version $y(t)$ of $x^{\prime}(t)$ by $x^{\prime}(t)$ itself.

Proposition 4. For almost all $\omega, x(t)$ is absolutely continuous in $t$ and $d x(t) / d t=x^{\prime}(t)$ for almost all $t$.

Noticing that $x^{\prime}(t)$ and $x(t)$ are measurable in $(t, \omega)$, we have, by Proposition 2 and simple computations,

$$
\left\|\int_{s}^{t} x^{\prime}(\theta) d \theta-(x(t)-x(s))\right\|^{2}=0
$$

and so

$$
P\left(\int_{s}^{t} x^{\prime}(\theta) d \theta=x(t)-x(s)\right)=1
$$

for every pair $(s, t)$ fixed. Therefore

$$
P\left(\int_{s}^{t} x^{\prime}(\theta) d \theta=x(t)-x(s) \text { for rational } t, s\right)=1 .
$$

Since $\int_{s}^{t} x^{\prime}(\theta) d \theta$ and $x(t)-x(s)$ are both continuous in $t, s$, we have

$$
P\left(\int_{s}^{t} x^{\prime}(\theta) d \theta=x(t)-x(s) \text { for real } t, s\right)=1
$$

which proves our proposition. 
Proposition 5. For almost all $\omega, x(t)=0$ only on a $t$-set of Lebesgue measure 0 in $0<t<T$.

Let $e_{0}(\xi)$ be the indicator of the single point 0 . Then $e_{0}(x(t, \omega))$ is measurable in $(t, \omega), \int_{0}^{T} e_{0}(x(t, \omega)) d t$ is the Lebesgue measures of the $t$-set : $x(t, \omega)=0$ and

$$
E\left(\int_{0}^{T} e_{0}(x(t, \omega)) d t\right)=\int_{0}^{T} E\left(e_{0}(x(t, \omega)) d t=\int_{0}^{T} P(x(t)=0) d t=0,\right.
$$

because $x(t, \omega)$ is Gauss distributed.

By Proposition 4 and 5 we can apply Lemma 2 in Section 2 to the sample functions of our process.

$$
\begin{aligned}
E\left(N_{c}\right) & \leq E(N) \\
& \leq E\left(\lim _{\varepsilon \downarrow 0} \frac{1}{2 \varepsilon} \int_{0}^{T}\left|x^{\prime}(t)\right| e_{(-\varepsilon, \varepsilon)}(x(t)) d t\right) \\
& \leq \lim _{\varepsilon \downarrow 0} \frac{1}{2 \varepsilon} \int_{0}^{T} E\left[\left|x^{\prime}(t)\right| e_{(-\varepsilon, \varepsilon)}(x(t))\right] d t \\
& \quad \text { (by Lebesgue-Fatou's theorem) } \\
& =\lim _{\varepsilon \downarrow 0} \frac{T}{2 \varepsilon} E\left[\left|x^{\prime}(0)\right| e_{(-\varepsilon, \varepsilon)}(x(0))\right] .
\end{aligned}
$$

By Proposition 2, $\left(x(0), x^{\prime}(0)\right)$ is Gauss distributed with

$$
\begin{array}{cl}
E(x(0))=a, & E\left(x^{\prime}(0)\right)=0 \\
E\left((x(0)-a)^{2}\right)=r(0), & E\left(x^{\prime}(0)^{2}\right)=-r^{\prime \prime}(0) \\
E\left(x^{\prime}(0)(x(0)-a)\right)=r^{\prime}(0)=\int_{-\infty}^{\infty} i \lambda d F(\lambda)=0,
\end{array}
$$

and so we have

$$
\begin{aligned}
E & {\left[\left|x^{\prime}(0)\right| e_{(-\varepsilon, \varepsilon)}(x(0))\right] } \\
& =\frac{1}{2 \pi \sqrt{-r^{\prime \prime}(0) r(0)}} \int_{R^{2}} \int \exp \left[-\frac{(\xi-a)^{2}}{2 r(0)}-\frac{\eta^{2}}{-2 r^{\prime \prime}(0)}\right]|\eta| e_{(-\varepsilon, \varepsilon)}(\xi) d \xi d \eta \\
& =\frac{1}{\pi}\left(-\frac{r^{\prime \prime}(0)}{r(0)}\right)^{1 / 2} \int_{-\varepsilon}^{\varepsilon} \exp \left[-\frac{(\xi-a)^{2}}{2 r(0)}\right] d \xi .
\end{aligned}
$$

Thus we have

$$
E(N) \leq \frac{T}{\pi} \sqrt{-\frac{r^{\prime \prime}(0)}{r(0)}} \exp \left[-\frac{a^{2}}{2 r(0)}\right] .
$$


By Lemma 1 in Section 2, we have

$$
\begin{aligned}
E(N) & \geq E\left(N_{c}\right) \geq E\left[\lim _{p \rightarrow \infty} \sum_{q=1}^{2^{p}} c\left(x\left(\frac{(q-1) T}{2^{p}}\right), x\left(\frac{q T}{2^{p}}\right)\right)\right] \\
& =\lim _{p \rightarrow \infty} E\left[\sum_{q=1}^{2^{p}} c\left(x\left(\frac{(q-1) T}{2^{p}}\right), x\left(\frac{q T}{2^{p}}\right)\right)\right] \\
& =\lim _{p \rightarrow \infty} T \cdot \frac{2^{p}}{T} E\left[c\left(x(0), x\left(2^{-p} T\right)\right)\right] .
\end{aligned}
$$

If we can prove

$$
\lim _{t \downarrow 0} \frac{1}{t} E[c(x(0), x(t))] \geq \frac{1}{\pi} \sqrt{-\frac{r^{\prime \prime}(0)}{r(0)}} \exp \left[-\frac{a^{2}}{2 r(0)}\right]
$$

then it follows from (4) that

$$
E(N) \geq \frac{T}{\pi} \sqrt{-\frac{r^{\prime \prime}(0)}{r(0)}} \exp \left[-\frac{a^{2}}{2 r(0)}\right]
$$

which, combined with (3), completes the proof of our theorem in cace $\int_{-\infty}^{\infty} \lambda^{2} d F(\lambda)<\infty$.

By Proposition 2, $(x(0), x(t))$ is Gauss distributed with

$$
\begin{array}{lc}
E(x(0))=E(x(t))=a & \\
E\left((x(0)-a)^{2}\right)=E\left((x(t)-a)^{2}\right)=r(0) & (=\alpha) \\
E((x(0)-a)(x(t)-a))=r(t) & (=\beta)
\end{array}
$$

and so

$$
\begin{aligned}
& \frac{1}{t} E[c(x(0), x(t))] \\
& =\frac{1}{t} \iint_{\xi^{\eta}<0} \frac{1}{2 \pi \sqrt{\alpha^{2}-\beta^{2}}} \exp \left[-\frac{\alpha\left((\xi-a)^{2}+(\eta-a)^{2}\right)-2 \beta(\xi-a)(\eta-a)}{2\left(\alpha^{2}-\beta^{2}\right)}\right] d \xi d \eta \\
& =e^{-a^{2} / \alpha+\beta} \frac{1}{t} \int_{\xi_{\eta<0}} \int_{\frac{1}{2 \pi}} \frac{1}{2 \sqrt{\alpha^{2}-\beta^{2}}} \exp \left[-\frac{\alpha\left(\xi^{2}+\eta^{2}\right)-2 \beta \xi \eta}{2\left(\alpha^{2}-\beta^{2}\right)}+\frac{(\xi+\eta) a}{\alpha+\beta}\right] d \xi d \eta \\
& =e^{-a^{2} / \gamma} \frac{1}{t} \int_{|v / u|<1 / \bar{i} / \gamma} \frac{1}{2 \pi} \exp \left[-\frac{u^{2}+v^{2}}{2}+\frac{\sqrt{2} v a}{\sqrt{\gamma}}\right] d u d v \\
& =e^{-a^{2} / \gamma} \frac{1}{2 \pi} \int_{0}^{\infty} e^{-\rho^{2} / 2} \rho d \rho \frac{1}{t} \int_{|\tan \theta|<-v_{\bar{i} / \gamma}} \exp \left[\frac{\sqrt{2} \rho \sin \theta a}{\sqrt{\gamma}}\right] d \theta .
\end{aligned}
$$


Since $|\sin \theta| \leq|\tan \theta|$, we have

$$
\begin{aligned}
& \frac{1}{t} E[c(x(0), x(t))] \\
& \quad \geq \exp \left(-a^{2} / \gamma\right) \frac{1}{2 \pi} \int_{0}^{\infty} e^{-\rho^{2} / 2} \rho d \rho \exp \left(-\frac{\sqrt{2 \delta} \rho a}{\gamma}\right) \frac{4}{t} \arctan \sqrt{\frac{\delta}{\gamma}} .
\end{aligned}
$$

As $t \rightarrow 0$, we have

$$
\begin{aligned}
\frac{1}{t} \arctan \sqrt{\frac{\delta}{\gamma} \sim \frac{1}{t} \sqrt{\frac{\delta}{\gamma}}} & =\sqrt{\frac{r(0), \quad \delta \rightarrow 0, r(t)}{(r(0)+r(t)) t^{2}}} \\
& =\sqrt{\frac{2 r(0)-r(t)-r(-t)}{t^{2}}} \sqrt{\frac{1}{2(r(0)+r(t))}} \\
& \left.\rightarrow \frac{1}{2} \sqrt{\frac{-r^{\prime \prime}(0)}{r(0)}}, \quad \text { (notice } r(-t)=r(t)\right)
\end{aligned}
$$

and so

$$
\begin{aligned}
\frac{\lim _{t \downarrow 0} \frac{1}{t} E[c(x(0), x(t))]}{} & \geq \exp \left(-\frac{a^{2}}{2 r(0)}\right) \frac{1}{\pi} \sqrt{\frac{-r^{\prime \prime}(0)}{r(0)}} \int_{0}^{\infty} \exp \left(-\rho^{2} / 2\right) \rho d \rho \\
& =\frac{1}{\pi} \sqrt{\frac{-r^{\prime \prime}(0)}{r(0)}} \exp \left(-\frac{a^{2}}{2 r(0)}\right) .
\end{aligned}
$$

4. Proof of Theorem in case $\int_{-\infty}^{\infty} \lambda^{2} d F(\lambda)=\infty$

In this case

$$
\lim _{h \downarrow 0} \frac{r(h)-2 r(0)+r(-h)}{h^{2}}=-\int_{-\infty}^{\infty} \lambda^{2} d F(\lambda)=-\infty .
$$

Therefore it is enough to prove

$$
E(N)=E\left(N_{c}\right)=\infty .
$$

By virtue of the continuity of the sample paths, we can apply Lemma 1 to get

$$
N \geq N_{c} \geq \lim _{p \rightarrow \infty} \sum_{q=1}^{2^{p}} c\left(x\left(\frac{(q-1) T}{2^{p}}\right), x\left(\frac{q T}{2^{p}}\right)\right)
$$

and so, using the same arguments as in the last part of Section 3, we get 


$$
\begin{aligned}
& E(N) \geq E\left(N_{c}\right) \geq E\left[\lim _{p \rightarrow \infty} \sum_{q=1}^{2^{p}} c\left(x\left(\frac{(q-1) T}{2^{p}}\right), x\left(\frac{q T}{2^{p}}\right)\right)\right] \\
& =\lim _{p \rightarrow \infty} T \cdot \frac{2^{p}}{T} E\left[c\left(x(0), x\left(2^{-p} T\right)\right)\right] \\
& \geq \frac{2 T}{\pi} \lim _{t \downarrow 0} \sqrt{\frac{2 r(0)-r(t)-r(-t)}{t^{2}}} \sqrt{\frac{1}{2(r(0)+r(t))}}=\infty \text {. }
\end{aligned}
$$

Department of Mathematics, Kyoto University and

Department of Mathematics, Stanford University

\section{REFERENCES}

[1] S. O. Rice : Mathematical analysis of random noise. Bell System Tech J. 24 (1945), 46-156.

[2] G. Hunt : Random Fourier transforms. Trans. Amer. Math. Soc. 71 (1956), 38-69.

[3] U. Grenander and M. Rosenblatt : Statistical analysis of stationary time series. New York, 1957.

[4] M. Kac: On the average number of real roots of a random algebraic equation. Bull. Amer. Math. Soc. 49 (1943), 314-319.

[5] V. A. Ivanov: On the average number of crossings of a level by sample functions of a stochastic processes. Th. Prob. Appl. 5 (1960), 319-323 (English translation).

[6] E. V. Bulinskaya : On the mean number of crossings of a level by a stationary Gaussian process. Th. Prob. Appl. 6 (1961), 435-438 (English translation). 